\title{
AVALIAÇÃO DE CICLO DE VIDA DO FERRO GUSA PRODUZIDO POR DIFERENTES LITERATURA REVISÃO SISTEMÁTICA DE
}

Adriano Souza Leão¹, Arilma do Carmo Tavares², Henrique Leonardo Maranduba³, Edna dos Santos Almeida ${ }^{2}$

${ }^{1}$ Centro Universitário SENAI CIMATEC, Avenida Orlando Gomes 1845, Salvador-BA; adrianoleaoeng@outlook.com

${ }^{2}$ Centro Universitário SENAI CIMATEC, Avenida Orlando Gomes 1845, Salvador-BA

${ }^{3}$ Instituto Federal de Educação, Ciência e Tecnologia - Campus Jequié, Jequié-BA

Resumo: O setor siderúrgico de ferro e aço tem sofrido pressões e transformações determinantes nos últimos anos haja vista seus impactos ambientais. A produção de ferro e aço é intensiva em energia e emissão de gases de efeito estufa. No entanto, no âmbito da ecologia industrial e economia circular, rotas alternativas emergentes tem apontado um potencial de performance ambiental superior. A Avaliação de Ciclo de Vida (ACV) é uma ferramenta reconhecida internacionalmente na academia e na indústria por possibilitar a mensuração e comparação do perfil ambiental de produtos e serviços. Neste estudo, realizou-se uma avaliação sistemática da literatura que concerne à questão dos impactos ambientais do ciclo de vida da produção de ferro gusa com o intuito de elencar as contribuições científicas e evidências existentes, e identificar lacunas. Os resultados da busca foram selecionados, classificados e analisados. Dentre os 15 trabalhos mais aderentes, não foi encontrada resposta completa às questões de pesquisa e lacunas a serem preenchidas foram identificadas.

Palavras-Chave: Impacto Ambiental; Ecologia Industrial; Alto-Forno; Ferro Primário

\section{LIFE CYCLE ASSESSMENT OF PIG IRON PRODUCED BY DIFFERERNT TECHNOLOGIES: SYSTEMATIC LITERATURE REVIEW}

\begin{abstract}
The steel and iron industry has been suffering major pressures and transformations in recent years given its environmental impacts. The production of iron and steel is intensive in energy use and greenhouse gas emitting. However, in the context of industrial ecology and circular economy, emerging alternative routes have pointed to a potential enhanced environmental performance. Life Cycle Assessment (LCA) is an internationally recognized tool in both the academia and the industry for enabling the measurement and comparison of the environmental features of products and services. In this study, a systematic review of the literature on the environmental impacts of the pig iron production life cycle was made to list the scientific contributions and existing evidence, and to identify gaps. The search results were selected, classified and assessed. Among the 15 most adherent manuscripts, no complete answer to the research questions was found and gaps to be filled were identified.
\end{abstract}

Keywords: Environmental Impact; Industrial Ecology; Blast Furnace; Hotmetal 


\section{INTRODUÇÃO}

O ferro gusa é o produto da redução de minério de ferro por um agente redutor carbonoso, também fonte de energia do processo [1]. Essa redução se processa em fornos industriais com auxílio de fluxantes e outros materiais. O gusa é produzido no mundo inteiro majoritariamente via alto-fornos [2]. A maior parte ( 70\%) é consumida na produção de aço. A composição mássica típica do ferro gusa possui pelo menos 92\% de ferro (Fe), 3,5-4,5\% de carbono $(\mathrm{C})$, podendo conter silício (Si), enxofre (S), fósforo $(\mathrm{P})$ e manganês $(\mathrm{Mn})$ como impurezas [1].

De acordo com Relatório Estatístico Anual da Associação Mundial de Aço [3], em 2017, a produção mundial de gusa foi da ordem de 1,2 trilhões de toneladas. A China foi o país com maior produção, cerca de $61,4 \%$ desse total. Em sexto lugar, o Brasil com 2,4\%. Segundo o Anuário Estatístico do Setor Metalúrgico [4], 32,1 milhões de toneladas foram produzidas no Brasil, sendo 72,6\% em Minas Gerais, $14,5 \%$ no Maranhão e Pará juntos, 9,1\% no Espírito Santo e 3,8\% no Mato Grosso do Sul.

Diante do cenário global de mudanças no mercado de matérias-primas, alternativas economicamente sustentáveis para siderurgia tem sido desenvolvidas [5]. Em face à pressão das mudanças climáticas no setor, a redução do consumo de energia, bem como das emissões de Gases de Efeito Estufa (GEE) estão na agenda. A produção de ferro e aço está entre as mais intensivas em uso de energia e corresponde à maior parcela de emissão de $\mathrm{CO}_{2}$ do setor manufatureiro, aproximadamente $27 \%$ [6]. Uma gama de tecnologias de baixo consumo de energia e de emissão aplicáveis à indústria siderúrgica já estão em estado de comercialização. Todavia, a informação sobre as tecnologias ainda não comercializadas é limitada.

De acordo com relatório do Centro de Gestão e Estudos Estratégicos [7], na discussão acerca do "desenvolvimento sustentável" no setor siderúrgico brasileiro são preocupações centrais, dentre outras, a normalização cada vez mais restritiva no tocante à exploração de recursos naturais e fortalecimento das ações das organizações ambientais. Estrategicamente, o setor caminha para articular-se aderindo à programas ambientais e metas de redução de GEE voluntários.

Recentemente, os mais novos altos-fornos tem sido aprimorados com foco nas matérias-primas de carregamento e injeção de combustíveis auxiliares [2]. Isso pode tornar o processo mais fluido, aumentando produtividade e, ao mesmo tempo, reduzindo a demanda de agentes redutores. O potencial de uso de briquetes se apresenta como alternativa para explorar resíduos de ferro e carbono no processo. Além disso, a reestruturação da matriz energética do setor, priorizando fontes renováveis, também é uma medida relevante para o avanço em simbiose industrial e economia circular.

Uma das ferramentas de análise ambiental de produtos e serviços da indústria é a Avaliação do Ciclo de Vida (ACV), metodologia internacionalmente reconhecida [8]. O método consiste em compilar as entradas e saídas de recursos materiais e energéticos do início ao final da vida de um sistema de produto e estimar os impactos produzidos sobre o meio ambiente [9]. Em virtude da sua padronização, é possível estabelecer comparações entre os sistemas produtivos (benchmarking ecológico).

Diante do exposto, este trabalho se propõe a investigar o estado da arte da avaliação de ciclo de vida ambiental da produção de ferro gusa através de uma revisão sistemática da literatura. 


\section{METODOLOGIA}

Realizou-se uma revisão sistemática da literatura para levantar contribuições científicas e evidências existentes, e identificar lacunas no que diz respeito ao problema de pesquisa anteposto. A estratégia usada foi buscar trabalhos em repositórios científicos concernentes ao tema e selecionar os manuscritos mais relevantes para posterior análise e discussão de seus principais aspectos e constatações.

As ações foram realizadas na seguinte sequência: sistematização de questões de pesquisa; definição dos descritores e período/ano de publicação; filtragem de trabalhos após leitura de título e resumo; seleção após leitura completa dos manuscritos disponíveis; identificação dos achados que respondem parcial ou totalmente às perguntas; e, por fim, análise crítica das publicações remanescentes.

O problema de pesquisa foi sistematizado em uma questão central (Q1) e em duas outras questões complementares (Q2 e Q3) para melhor delimitação do problema, oriundas da contextualização do referencial teórico, como segue:

Q1. Existem trabalhos que aplicaram a ACV para avaliar o perfil ambiental do ferro gusa produzido por pelo menos uma rota/tecnologia?

Q2. Existem trabalhos que estimam a pegada de carbono da produção de ferro gusa?

Q3. Existem trabalhos que estimam a demanda acumulada de energia da produção de ferro gusa?

As questões complementares remetem não apenas aos impactos operacionais do ciclo de vida produtivo do ferro gusa, emissões diretas e eficiência energética na etapa de manufatura/produção dentro da usina, mas também, em uma perspectiva mais abrangente, às etapas pré-operacionais (pegada de carbono e demanda acumulada de energia). Essas últimas são categorias de impacto da ACV que compreendem contribuições desde a extração de matérias-primas, pré-manufatura dos principais insumos demandados no processo, até o portão de entrada da usina.

Pesos foram atribuídos para cada questão em função da aderência do manuscrito. No caso de resposta completa à questão Q1, a publicação recebe nota 5, e resposta parcial, nota 2,5. Para Q2, total 3 e parcial 1,5. E para Q3, total 2 e parcial 1. Um manuscrito pode atingir a nota máxima de 10, sendo que quanto mais aderente 0 conteúdo do trabalho, mais sua nota se aproxima desse limite.

Sinônimos e termos alternativos foram considerados como em "ferro gusa" e "siderurgia". Da mesma forma, acrônimos/abreviaturas como em "ACV" e "Avaliação de Ciclo de Vida". Além desses, o asterisco $\left({ }^{*}\right)$ para contemplar diferentes sufixos, como em "ambient" que é interpretado pelos mecanismos de busca como o conjunto "ambiente", "ambiental", "ambientais" e outros. Foram usados os descritores a seguir:

- Ferro gusa, Siderurgia, ACV, Avaliação de Ciclo de Vida, Eficiência energética, Carbono, Ambiental;

- Pig iron, Ironmaking, LCA, Life Cycle Assessment, Energy efficiency, Carbon, Environmental.

O string foi montado contendo três elementos principais separados pelo booleano "AND". O primeiro é composto pelos descritores referentes ao objeto de 
estudo ("ferro gusa" OR siderurgi*). O segundo, pelo método científico de análise de impacto ambiental (acv or "avaliação de ciclo de vida"). E o terceiro, pelos termos auxiliares ("eficiência energética" OR carbon* OR ambient*). Descritores do objeto de estudo e do método são referentes à questão principal $(\mathrm{Q} 1)$ e os auxiliares, às complementares (Q2 e Q3). O mesmo procedimento foi feito com os descritores em inglês.

A busca foi realizada nos repositórios Web of Science, Science Direct, Scopus e Scielo, bem como no Scholar Google. O período considerado para o estado da arte foram os últimos 3 anos e o ano corrente, de 2016 a 2019. Publicações relevantes dos anos anteriores, de 2000 a 2015, também foram consideradas. Os critérios de inclusão que geram exclusão utilizados foram aplicados na seguinte ordem:

- Descritores contidos no título, resumo e/ou palavras-chave;

- Título, resumo e palavras-chave aderentes ao tema;

- Acesso ao documento completo (visualização ou download);

- Metodologia, resultados, discussão e conclusões aderentes às questões;

- Confronto com as questões de pesquisa e atribuição dos pesos.

Após isso, os trabalhos remanescentes de todo o processo de seleção foram analisados um a um, com destaque para os de maior nota, elencando seus principais aspectos, contribuições e lacunas tendo em vista o objetivo desta pesquisa.

\section{RESULTADOS E DISCUSSÃO}

As buscas foram realizadas no dia 05 de agosto de 2019. A Tabela 1 mostra o número de publicações encontradas na primeira busca utilizando termos em inglês e português para o período considerado o estado da arte e também para o anterior. Nota-se que a maioria dos manuscritos foi encontrada no Scholar Google (62\%). Já Scopus, Web of Science e Science Direct correspondem à 38\% dos achados na primeira etapa. No Scielo, nenhum trabalho foi recuperado. A Tabela 2 apresenta os resultados após verificação e seleção por título, resumo e palavras-chave.

Tabela 1. Primeira etapa: número de trabalhos encontrados nas buscas

\begin{tabular}{lcccccc}
\hline \multirow{2}{*}{ Repositório } & \multicolumn{6}{c}{ Com descritores em inglês / português } \\
& $\mathbf{2 0 1 9}$ & $\mathbf{2 0 1 8}$ & $\mathbf{2 0 1 7}$ & $\mathbf{2 0 1 6}$ & $\mathbf{2 0 0 0 - 2 0 1 5}$ & Total \\
\hline Scholar Google & $6 / 0$ & $9 / 3$ & $7 / 4$ & $9 / 2$ & $33 / 15$ & 88 \\
Scopus & $0 / 0$ & $2 / 0$ & $0 / 0$ & $1 / 0$ & $21 / 0$ & 24 \\
Web of Science & $0 / 0$ & $2 / 0$ & $0 / 0$ & $1 / 0$ & $19 / 0$ & 22 \\
Science Direct & $0 / 0$ & $1 / 0$ & $0 / 0$ & $0 / 0$ & $7 / 0$ & 8 \\
Scielo & $0 / 0$ & $0 / 0$ & $0 / 0$ & $0 / 0$ & $0 / 0$ & 0 \\
\hline
\end{tabular}

Tabela 2. Segunda etapa: número de trabalhos remanescentes após leitura de título, resumo e palavras-chave

\begin{tabular}{|c|c|c|c|c|c|c|c|c|}
\hline \multirow{2}{*}{ Repositório } & \multicolumn{6}{|c|}{ Com descritores em inglês / português } & \multirow{2}{*}{$\begin{array}{l}\text { Indispo- } \\
\text { níveis }\end{array}$} & \multirow{2}{*}{$\begin{array}{l}\text { Dupli- } \\
\text { cados }\end{array}$} \\
\hline & 2019 & 2018 & 2017 & 2016 & $2000-2015$ & Total & & \\
\hline Scholar Google & $2 / 0$ & $4 / 0$ & $5 / 0$ & $5 / 0$ & $9 / 2$ & 27 & 6 & \\
\hline Scopus & $0 / 0$ & $0 / 0$ & $0 / 0$ & $0 / 0$ & $14 / 0$ & 14 & 1 & \\
\hline Web of Science & $0 / 0$ & $0 / 0$ & $0 / 0$ & $0 / 0$ & $16 / 0$ & 16 & 1 & 17 \\
\hline Science Direct & $0 / 0$ & $0 / 0$ & $0 / 0$ & $0 / 0$ & $6 / 0$ & 6 & 0 & \\
\hline Scielo & $0 / 0$ & $0 / 0$ & $0 / 0$ & $0 / 0$ & $0 / 0$ & 0 & 0 & \\
\hline
\end{tabular}


Dos 71 trabalhos selecionados, 8 não estavam disponíveis para leitura completa e 17 eram duplicados, isto é, encontrados em mais de um repositório (Fig. 1). Um dos achados é um livro, de onde 2 capítulos foram selecionados. Isso resultou em uma soma de 47 trabalhos diferentes para serem avaliados, incluindo artigos de periódico, artigos de congresso, capítulos de livro, dissertações, teses e relatórios técnicos.

Um número substancial de publicações duplicadas (94\%) foi encontrado entre os repositórios Scopus, Web of Science e Science Direct. Já o Google Scholar, apesar de seu mecanismo de busca ser muito abrangente, apenas 1 trabalho era duplicado em relação às outras bases. Embora tenha sido o repositório com maior quantidade de trabalhos recuperados até esta etapa, 22\% não estavam disponíveis para visualização ou download.

Figura 1. Trabalhos selecionados até etapa 2 por repositório e por disponibilidade

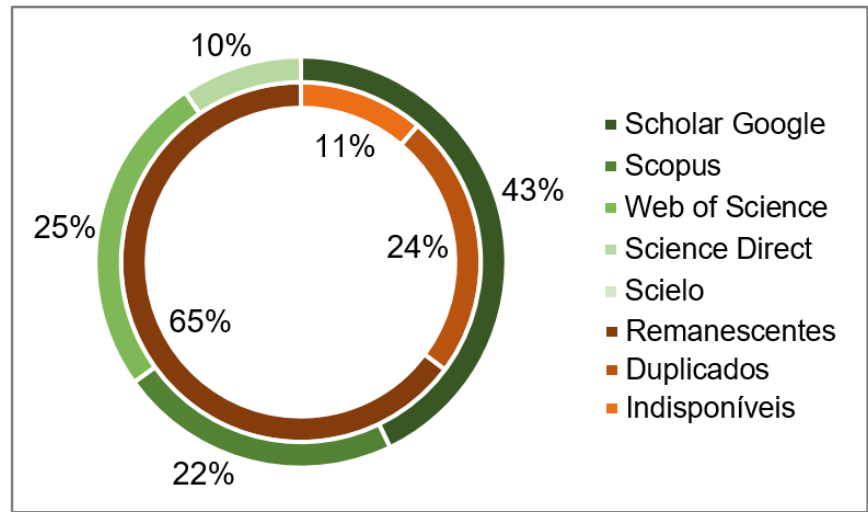

A maior parte dos artigos (papers) publicados de 2017 para trás estava disponível. Outros mais recentes não estavam disponíveis, provavelmente, por serem de acesso exclusivo dos assinantes dos periódicos. As taxas de submissão custeiam, entre outros, avaliação por pares e editoração. Artigos submetidos na modalidade gratuita normalmente passam a ter acesso livre após dois anos, procedimento comum nas principais editoras de artigos científicos do mundo e revistas de alto impacto.

$\mathrm{Na}$ terceira etapa, as publicações com visualização ou download permitido dentro do VPN concedido ao SENAI CIMATEC pela CAPES foram analisadas, permanecendo apenas as consideradas contenedoras de respostas no mínimo parciais para pelo menos uma das questões de pesquisa, sendo pontuadas de acordo. O Quadro 1 no Apêndice A do Material Suplementar apresenta informação completa dos manuscritos retidos após esta etapa. A Tabela 3 mostra os 37 trabalhos resultantes.

Foram encontradas 36 publicações que respondem às perguntas levantadas, sendo 15 do estado da arte e 21 do período anterior. Houve mais respostas parciais $(61 \%)$ que totais (39\%). Somente 15 trabalhos (42\%) responderam à pergunta central da pesquisa (Q1), sendo 11 de forma total, mas com lacunas. Foram encontradas 36 respostas para a questão Q2, e 17 para a Q3. De modo geral, a maioria das respostas para Q2 e Q3 foi parcial, visto que em alguma medida houve análise de emissões diretas de $\mathrm{CO}_{2}$ ou GEE ou ainda a eficiência energética do processo, que são uma parte mais restrita do ciclo de vida procurado nesta pesquisa, que inclui também pegada de carbono e demanda energética acumulada nas etapas pré-operacionais. 
Tabela 3. Terceira etapa: confronto com perguntas após leitura completa dos disponíveis

\begin{tabular}{|c|c|c|c|c|c|c|c|c|c|c|}
\hline \multirow{2}{*}{$\begin{array}{l}\text { Publi- } \\
\text { cação }\end{array}$} & \multirow{2}{*}{ Ano } & \multirow{2}{*}{ Autor(es) } & \multirow{2}{*}{$\begin{array}{l}\text { País filiação } \\
1^{\circ} \text { autor }\end{array}$} & \multirow{2}{*}{$\begin{array}{l}\text { Tipo de } \\
\text { publicação }\end{array}$} & \multicolumn{3}{|c|}{ Pergunta } & \multicolumn{2}{|c|}{ Reposta } & \multirow{2}{*}{ Nota } \\
\hline & & & & & Q1 & Q2 & Q3 & Total & Parcial & \\
\hline P1 & 2019 & LV, Sun e Su & China & Art. periódico & & $x$ & $x$ & & $x$ & 2,5 \\
\hline $\mathrm{P} 2$ & 2018 & Suopajärvi et al. & Finlândia & Art. periódico & & $x$ & & & $x$ & 1,5 \\
\hline P3 & 2018 & Chowdhury et al. & Inglaterra & Art. periódico & & & $x$ & & $x$ & 1,0 \\
\hline P4 & 2018 & Ma, Eneji e Wu & China & Art. periódico & & & $x$ & & $x$ & 1,0 \\
\hline P5 & 2018 & Miah & Inglaterra & Tese & $x$ & $x$ & $x$ & & $x$ & 5,0 \\
\hline P6 & 2017 & Suopajarvi et al. & Finlândia & Art. periódico & & $x$ & & & $x$ & 1,5 \\
\hline P7 & 2017 & Shatokha, Tymoshenko e Semenko & Ucrânia & Capítulo livro & & $x$ & $x$ & & $x$ & 2,5 \\
\hline P8 & 2017 & Shatokha & Ucrânia & Capítulo livro & & $x$ & $x$ & & $x$ & 2,5 \\
\hline P9 & 2017 & Cardenas et al & México & Art. periódico & & $x$ & $x$ & & $x$ & 2,5 \\
\hline P10 & 2017 & Bains, Psarrasa e Wilcox & EUA & Art. periódico & & $x$ & & & $x$ & 1,5 \\
\hline P11 & 2016 & Mousa et al. & Suécia & Art. periódico & & $x$ & $x$ & & $\mathrm{x}$ & 2,5 \\
\hline P12 & 2016 & Pan et al. & China & Art. periódico & & & $x$ & & $x$ & 1,0 \\
\hline P13 & 2016 & Wu, Pu e Qi & China & Art. periódico & & $x$ & $x$ & & $x$ & 2,5 \\
\hline $\mathrm{P} 14$ & 2016 & Olmez et al. & Turquia & Art. periódico & $x$ & $x$ & & $x$ & & 8,0 \\
\hline P15 & 2016 & Bahadir & EUA & Art. periódico & $x$ & $x$ & $x$ & & $x$ & 5,0 \\
\hline P16 & 2015 & Saade, Silva e Gomes & Brasil & Dissertação & $x$ & $x$ & & $x$ & & 8,0 \\
\hline P17 & 2015 & Reuter,Schaik e Gediga & Finlândia & Art. periódico & $x$ & $x$ & & & $x$ & 4,0 \\
\hline P18 & 2015 & Jahanshahi et al. & Austrália & Art. periódico & & $x$ & & & $x$ & 1,5 \\
\hline P19 & 2015 & Crossin & Austrália & Art. periódico & & $x$ & & $x$ & & 3,0 \\
\hline P20 & 2015 & Chen et al. & China & Art. periódico & $x$ & $x$ & & $x$ & & 8,0 \\
\hline P21 & 2015 & Arasto & Finlândia & Tese & & $x$ & & & $x$ & 1,5 \\
\hline P22 & 2014 & Fick et al. & França & Art. periódico & $x$ & $x$ & & $x$ & & 8,0 \\
\hline P23 & 2014 & Suopajärvi, Pongrácz e Fabritius & Finlândia & Art. periódico & & $x$ & $x$ & $x$ & & 5,0 \\
\hline P24 & 2013 & Vadenbo, Boesch e Hellweg & Suíça & Art. periódico & $x$ & $x$ & & $x$ & & 8,0 \\
\hline P25 & 2013 & Choi & Noruega & Dissertação & $x$ & $x$ & & $x$ & & 8,0 \\
\hline P26 & 2013 & Saade & Brasil & Dissertação & $x$ & $x$ & & $x$ & & 8,0 \\
\hline P27 & 2013 & Korol & Polônia & Art. periódico & $x$ & $x$ & $x$ & & $x$ & 5,0 \\
\hline P28 & 2012 & Fick et al. & França & Art. congresso & $\hat{x}$ & $\hat{x}$ & & $x$ & & 8,0 \\
\hline P29 & 2012 & Korol & Polônia & Art. congresso & $x$ & & $x$ & $x$ & & 7,0 \\
\hline P30 & 2011 & Ding e Li & China & Art. congresso & $x$ & $x$ & & $x$ & & 8,0 \\
\hline P31 & 2011 & Kuramochi & Japão & Tese & & $x$ & & & $x$ & 1,5 \\
\hline P32 & 2011 & Ramos & Inglaterra & Tese & & $x$ & $x$ & & $x$ & 2,5 \\
\hline P33 & 2009 & Norgate e Langberg & Austrália & Art. periódico & & $x$ & & $x$ & & 3,0 \\
\hline P34 & 2008 & Sablowski & Brasil & Tese & $x$ & $x$ & & $x$ & & 8,0 \\
\hline P35 & 2007 & Ryman & Suécia & Dissertação & & $x$ & $x$ & & $x$ & 2,5 \\
\hline P36 & 2001 & Gielen e Moriguchi & Japão & Relat. técnico & & $x$ & $x$ & & $x$ & 2,5 \\
\hline
\end{tabular}

A Figura 2 exibe o perfil do problema de pesquisa da amostra final de publicações por categoria. Verificou-se uma tendência de crescimento do número de estudos a partir de 2011, com destaque para o ano de 2015, mas também nos 3 anos subsequentes (Fig. 2a). Notou-se que, na maior parte dos achados, o primeiro autor tinha apenas 1 publicação concernente ao tema em específico. Entretanto, 5 dos 30 autores contribuíram 2 vezes ou mais, representando 25\% da amostra (Fig. 2b).

Observou-se maior ocorrência de documentos mais longos (teses, dissertações e relatórios) no período anterior ao estado da arte, e mais artigos de periódicos em pesquisas recentes, tratando as questões de forma mais pontual. Cerca de $58 \%$ dos achados são artigos de periódicos, demonstrando a relevância do tema como objeto de discussão científica em revistas de alto impacto (Fig. 2c). Do restante, 25\% são teses ou dissertações e 17\% artigos de congresso, capítulos de livro ou relatório. Os países de filiação do primeiro autor com maior produção são a China com 17\%, seguido da Finlândia com 14\%, e Brasil, Austrália e Inglaterra empatados com $8 \%$ (Fig. 2d). Os $44 \%$ restantes estão divididos entre países americanos e europeus. 


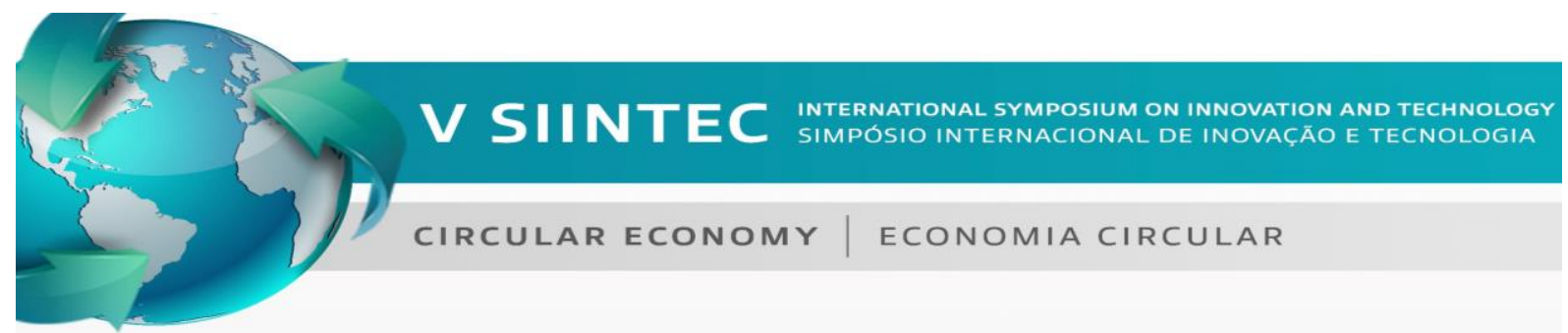

Nenhuma das publicações atingiu nota máxima. No entanto, boa parte (28\%) alcançou nota 8,0 demonstrando que os critérios das primeiras etapas de seleção foram assertivos e que as questões de pesquisa estão, de fato, relacionadas (Fig. 2e). Os achados com nota final entre 5,0 e 7,0 representam $14 \%$. Juntos com os de nota 8,0 somam 15 estudos ( $42 \%$ da amostra), representando aqueles com maior aderência cujos resultados e constatações terão maior influência na análise que segue. Os outros 58\% alcançaram notas entre 1,5 e 4,0.

Figura 2. Perfil do problema de pesquisa por categoria de análise

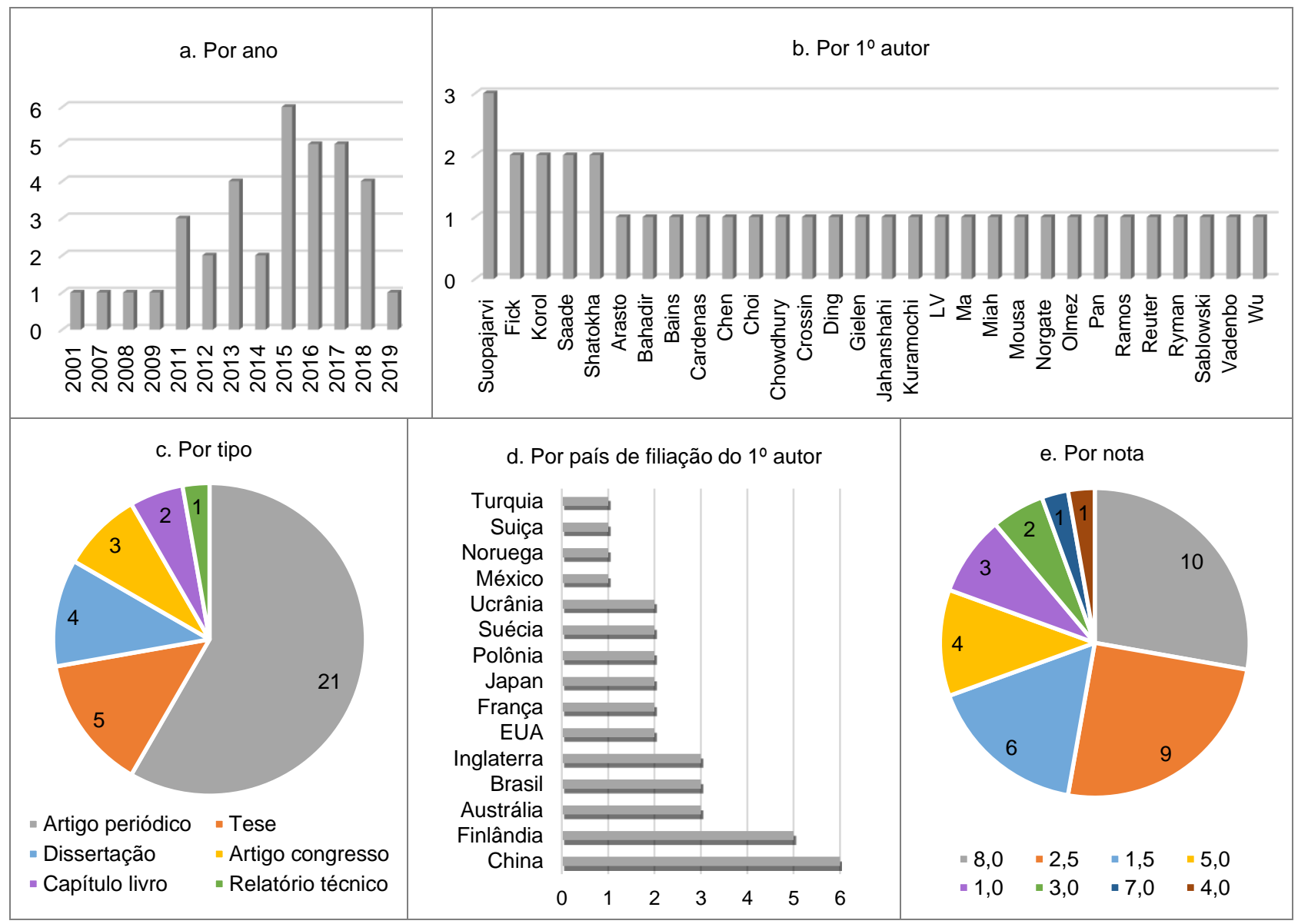

Parte considerável dos trabalhos foi de natureza primária, isto é, investigações experimentais ou teóricas que produziram evidências sobre redução da pegada de carbono, captura e armazenamento de carbono, aumento da eficiência energética, no contexto da indústria siderúrgica mundial ou local referentes ao país nacionalidade dos autores. Porém, alguns propuseram estudos secundários, revisões de tecnologias disponíveis e estado da arte, envolvendo também questões políticas e regulatórias.

As abordagens mais comuns se pautaram na análise de matérias-primas mais renováveis ou alternativas tecnológicas pontuais e incrementais para melhoria da eficiência energética e/ou redução de emissões da principal rota, o alto-forno, quase sempre associada ao ciclo de vida do aço. Das tecnologias alternativas, apenas o COREX ${ }^{\circledR}$ foi objeto de comparação com o alto-forno, não havendo sido encontradas 
análises dessa natureza sobre outras rotas emergente de produção de gusa. São tecnologias emergentes Finex ${ }^{\circledR}$, Midrex ${ }^{\circledR}$, Hlsmelt $^{\circledR}$, ITmk $^{\circledR}{ }^{\circledR}$, Tecnored ${ }^{\circledR}$ e outros [5].

No que concerne ao estudo de matérias-primas do processo, apenas 1 trabalho considerou alternativas de fonte de ferro, como resíduos, sucata, carepa e outros. A maioria predominante teve foco nas fontes de carbono, agente redutor ao mesmo tempo combustível. Em alguns casos, citou-se a esgotamento de recursos a partir de estimativas de escassez de fontes de combustíveis fósseis, por exemplo. Não foram resultados e discussões sobre danos ao ecossistema ou saúde humana.

Sabe-se que pegada de carbono é a categoria de impacto em maior importância para indústria nos dias em curso. Todavia, não deve ser a única na avaliação do perfil ambiental de um produto ou serviço industrial. Além disso, menções ou atendimento às diretrizes da regra de categoria de produto para análises do setor de ferro e aço não foram encontradas. Dos que conduziram uma ACV, apenas 5 estudos avaliaram 4 ou mais categorias de impacto, a fim de gerar um perfil ambiental mais completo para constatações mais robustas e consistentes.

Apesar de eficiência energética ser uma grandeza técnica, possui implicações ambientais indiretas e indissociáveis, e por isso passou a ser considerada como uma categoria impacto. Nessa perspectiva, a pegada energética não deve refletir apenas aspectos e impactos da etapa de operação ou uso, mas também de forma cumulativa agregando contribuições das etapas pré-operacionais do ciclo de vida analisado, incluindo o conteúdo renovável e não-renovável de suas fontes.

\section{CONCLUSÃO}

Observou-se que os estudos de avaliação do ciclo de vida da produção de ferro gusa por diferentes rotas é limitado em número e completeza, embora pesquisas relacionadas tenham aumentado em número e relevância nos últimos anos. Dos 15 trabalhos de maior aderência, nenhum realizou uma ACV do ferro gusa considerando um número substancial de categorias de impacto a fim de montar um perfil ambiental robusto, ou tomou como base a regra de categoria de produto para siderurgia, ou mesmo agregou pegada de carbono e demanda acumulada de energia, dois aspectos relevantes evidenciados no referencial teórico. Diante disso, verificou-se que o objeto de estudo nas condições colocadas ainda é, portanto, uma lacuna científica a ser explorada, principalmente no que diz respeito às tecnologias alternativas emergentes e as reivindicações de eficiência ambiental superior.

\section{Agradecimentos}

Agradecimento especial a EMBRAPII, que patrocinou a bolsa de mestrado referente ao projeto Curtain Wall - Tecnored para o pesquisador/autor deste artigo.

\section{Material suplementar}

APÊNDICE A - Quadro A1 


\section{REFERÊNCIAS}

${ }^{1}$ International Iron Metallics Association (IIMA). Pig Iron: Overview. 2018.

${ }^{2}$ SMIL, V. Modern Ironmaking and Steelmaking: Furnaces, Processes, and Casting. In. CAVALIERE, P. Ironmaking and Steelmaking Processes: Greenhouse Emissions, Control, and Reduction. 2016.

${ }^{3}$ World Steel Association (WSA). Statistics: Crude Steel Production. 2018.

${ }^{4}$ Ministério de Minas e Energia (MME). Anuário Estatístico do Setor Metalúrgico. 2018.

${ }^{5}$ GORDON, Y.; KUMAR, S.; FREISLICH, M.; YAROSHENKO, Y.; SPIRIN, N. The Modern Technology of Iron and Steel Production and Possible Ways of their Development Introduction. Steel in Translation. 2015.

${ }^{6}$ HASANBEIGI, A.; PRICE, L.; ARENS, M. Emerging Energy-efficiency and Carbon Dioxide Emissions-reduction Technologies for the Iron and Steel Industry. Berkeley National Laboratory. 2013.

${ }^{7}$ Centro de Gestão e Estudos Estratégicos (CGEE). Siderurgia no Brasil: 20102025. 2010.

${ }^{8}$ Associação Brasileira de Normas Técnicas (ABNT). NBR ISO 14040: Gestão ambiental - Avaliação do Ciclo de Vida - Princípios e Estrutura. 2009.

${ }^{9}$ Instituto Brasileiro de Informação em Ciência e Tecnologia (IBICT). Desenvolvimento Sustentável e Avaliação de Ciclo de Vida. 2014. 